\title{
Rehabilitation and Return to Sport After Medial Patellofemoral Complex Reconstruction
}

\author{
Rachel E. Lampros, P.T., D.P.T., S.C.S., Ashley L. Wiater, P.T., D.P.T., S.C.S., and \\ Miho J. Tanaka, M.D.
}

\begin{abstract}
The medial patellofemoral complex (MPFC) consists of the medial patellofemoral ligament and medial quadriceps tendon femoral ligament, which play a critical role stabilizing the patella against lateral translation. After a patellar dislocation, athletes with recurrent dislocations have functional limitations that may limit their return to their prior level of competition, requiring surgical reconstruction. Although ample literature exists delineating return-to-play (RTP) considerations after anterior cruciate ligament reconstruction, there is a paucity of evidence specific to MPFC reconstruction. Athletes aiming to return to sport after MPFC reconstruction require the same methodical treatment approach to ensure safe RTP. A criterion-based periodical assessment of progress that measures range of motion, strength, neuromuscular control, balance, agility, and power are pivotal components of rehabilitating this population. A combination of objective and subjective criteria should be assessed when determining an individual's readiness for sports-specific activities. A battery of functional tests, including quadriceps strength testing, single-limb hop testing, lateral step-down test, the lateral leap and catch test, the Y-balance test, and the depth jump should be considered when evaluating the athlete for readiness for sport, incorporating specific understanding of the biomechanics of the patellofemoral joint. We discuss the considerations for return-to-sport rehabilitation and testing after MPFC reconstruction, to provide clinicians working with an athletic population a framework to adequately prepare their athletes for safe return to sport.
\end{abstract}

\section{Introduction}

$\mathbf{P}$ atellar instability can be a complex injury to manage in the athletic population. The majority of acute patellar dislocations occurs in the lateral direction and can be a season-ending injury in some athletes. Fifty to $60 \%$ of first-time lateral patellar dislocations occur during sports-related physical activity, with 93\% of these dislocations being the result of noncontact play, where the athlete's foot is planted, with slight flexion of the knee, and a dynamic valgus and external rotation

Massachusetts General Hospital, Sports Physical Therapy, Boston, Massachusetts, U.S.A. (R.E.L., A.L.W.); and Massachusetts General Hospital, Harvard Medical School, Department of Orthopaedic Surgery, Boston, Massachusetts, U.S.A. (M.J.T.).

Full ICMJE author disclosure forms are available for this article online, as supplementary material.

Received August 1, 2021; accepted September 15, 2021.

Address correspondence to Dr. Miho J. Tanaka, M.D., Department of Orthopaedic Surgery, Massachusetts General Hospital, 175 Cambridge St., 4th Floor, Boston, MA 02114,U.S.A.E-mail:Mtanaka5@mgh.harvard.edu

(C) 2021 THE AUTHORS. Published by Elsevier Inc. on behalf of the Arthroscopy Association of North America. This is an open access article under the CC BY-NC-ND license (http://creativecommons.org/licenses/by-nc-nd/4.0/). 2666-061X/211092

https://doi.org/10.1016/j.asmr.2021.09.030 stress. ${ }^{1-3}$ Football, soccer, basketball, and gymnastics have been identified as high-risk sports for patella dislocation. $^{2}$

The annual incidence of acute lateral patellar dislocations has been reportedly as high as 29 cases per 100,000 person-year in those 10 to 17 years of age. ${ }^{1}$ Lateral dislocations occur more frequently in the younger athletic population, with individuals under the age of 20 demonstrating the highest risk. Skeletally immature, taller adolescent females also appear to have an increased risk when compared to their peers. ${ }^{1}$ Other morphological risk factors include patella alta, trochlear dysplasia, increased Q-angle or lateralized tibial tuberosity, genu valgum, ligamentous laxity, tibial torsion, and increased femoral anteversion. ${ }^{4}$

The medial patellofemoral ligament (MPFL) has been described as the primary static stabilizer of the patellofemoral joint. ${ }^{5,6}$ It provides static restraint against lateral patellar translation between 0 and 30 to $70^{\circ}$ of knee flexion. ${ }^{6,7}$ More recent studies have described that additional fibers extending to the quadriceps tendon exist to form the medial quadriceps tendon femoral ligament (MQTFL), which has led to the term medial patellofemoral complex (MPFC) to allow for the variability in attachment sites of this ligament. ${ }^{8-13}$ In cases 
Table 1. Early-Stage Rehabilitation Protocol after Medial Patellofemoral Complex Reconstruction

\begin{tabular}{|c|c|c|c|}
\hline & Phase 0 & Phase 1 & Phase 2 \\
\hline Optimal Time Frame & $0-2$ Weeks & 2-6 Weeks & 6-12 Weeks \\
\hline Criteria to progress & $\begin{array}{l}\text { Achieve full } \\
\text { passive knee } \\
\text { extension } \\
\text { - TTWB and } \\
\text { progress to WBAT } \\
\text { with brace locked } \\
\text { in extension for } \\
\text { ambulation }\end{array}$ & $\begin{array}{l}\text { Maintain full extension } \\
\text { - Achieve knee flexion of }>125 \\
\text { degrees } \\
\text { - Trace to no suprapatellar } \\
\text { effusion } \\
\text { - Good quadriceps activation } \\
\text { - WBAT with brace unlocked } \\
\text { - Normal gait mechanics } \\
\text { - Independent straight-leg raise } \\
\text { - Ascend 8-inch step with good } \\
\text { neuromuscular control }\end{array}$ & $\begin{array}{l}\cdot \text { Full ROM } \\
\cdot \text { No suprapatellar } \\
\text { effusion } \\
\cdot \text { Ambulate stairs with } \\
\text { normal mechanics } \\
\cdot \text { Hip abductor LSI } \\
>80 \% \\
\cdot \text { Hamstring LSI }>70 / \\
80 \% \\
\cdot \text { Good neuromuscular } \\
\text { control with step-down }\end{array}$ \\
\hline Exercise recommendations & $\begin{array}{l}\text { Straight leg raises, } \\
\text { flexion, } \\
\text { abduction, and } \\
\text { adduction } \\
\text { - Quad sets with } \\
\text { NMES } \\
\text { - Glute sets }\end{array}$ & $\begin{array}{l}\text { Gentle patellar mobilizations } \\
\text { - Quad isometrics at } 0^{\circ} \text { and } 90^{\circ} \\
\text { - Straight leg raises with } \\
\text { resistance } \\
\text { - Leg press } 90^{\circ}-40^{\circ} \\
\text { - Mini squats } 0^{\circ}-50^{\circ} \\
\text { - Progressive hamstring and hip } \\
\text { strengthening }\end{array}$ & $\begin{array}{l}\text { Patellar mobilizations } \\
\text { · Quadriceps stretching } \\
\text { · Isotonics } 90-40^{\circ} \\
\text { · Progressive resisted } \\
\text { quad strengthening } \\
\text { (Step-ups, lateral step- } \\
\text { downs, leg press, wall } \\
\text { slides) } \\
\text { · Progress SL balance } \\
\text { exercises } \\
\text { · Nonimpact } \\
\text { cardiovascular training } \\
\text { (bike, elliptical) }\end{array}$ \\
\hline
\end{tabular}

LSI, limb symmetry index; NMES, neuromuscular electrical stimulation; TTWB, toe touch weight bearing; ROM, range of motion; SL, single leg; WBAT, weight bearing as tolerated.

of recurrent patellar instability, reconstruction of the MPFC fibers are performed to recreate the medial restraint, which has been associated with improvements in clinical and biomechanical function.

MPFC surgery has been described in a variety of techniques and can consist of reconstruction of the MPFL or MQTFL or both. ${ }^{8,14,15}$ While the location of attachment sites, types of grafts, and fixation methods may vary, each reconstruction technique has been shown to restore patellar stability and knee function. ${ }^{8,14,16}$ One of the primary considerations during patellar stabilization surgery is the need for concurrent procedures to optimize patellar stability. Bony malalignment from tuberosity lateralization and patella alta have been associated with increased graft anisometry and have been shown to decrease the ability of the MPFL graft in restoring patellar kinematics. ${ }^{17,18}$ When additional procedures are performed to address such morphological abnormalities, such as tibial tuberosity osteotomy (TTO), the timing and progression of rehabilitation under surgery can be delayed. Our current discussion focuses on rehabilitation considerations for isolated soft tissue procedures, and those with additional bony procedures should undergo modifications to their protocols accordingly.

Overall return to play (RTP) rates after MPFC surgery are reported to be $85.1 \%$, with the rate of returning to the same level of play being lower at 68.3 , at an average time of 7.0 months. ${ }^{19}$ On average, RTS time frame is 6.7 months with a wide range of 3-12 months. ${ }^{20}$ Slower return-to-sport timelines are also seen with higher levels of activity and concomitant procedures, such as tibial tubercle osteotomy (TTO) and lateral retinacular release. ${ }^{21}$ After patellar stabilization surgery, proper recovery of function is more important than time-based criteria for RTS. ${ }^{22}$ On the basis of a thorough understanding of the biomechanics of the patellofemoral joint, we discuss postoperative considerations after MPFC reconstruction and provide clinical recommendations pertaining to RTS guidelines in this population.

\section{Rehabilitation after MPFC Reconstruction}

A complete understanding the arthrokinematics of the patellofemoral joint is a critical component of rehabilitating this population. Closed kinetic chain exercises (CKC) have been consistently shown to result in less patellofemoral joint reaction force from 0 to $50^{\circ}$ of flexion, whereas open kinetic chain (OKC) exercises demonstrate less compressive forces from 50 to 90 degrees of flexion. ${ }^{23-25}$ Furthermore, OKC exercises have been shown to generate significantly greater patellofemoral joint compressive forces at flexion angles less than $60^{\circ}$, and CKC exercises generate significantly greater compressive forces at flexion angles greater than $85^{\circ}$. 
Table 2. Late-Stage Rehabilitation Protocol after Medial Patellofemoral Complex Reconstruction

\begin{tabular}{|c|c|c|c|}
\hline & Phase 3 & Phase 4 & Phase 5 \\
\hline Optimal time frame & 12-16 Weeks & 16-20 Weeks & $20+$ Weeks \\
\hline Criteria to progress & $\begin{array}{l}\text { Quadriceps LSI >80\% } \\
\text { · Hip abductors LSI }>90 \% \\
\text { · Hamstring LSI }>80 / 90 \% \\
\text { · Good neuromuscular control } \\
\text { with } 60 \text {-second timed step-down } \\
\text { test } \\
\text { · Good landing mechanics with 60- } \\
\text { second timed lateral leap and catch } \\
\text { · Good landing mechanics with } \\
\text { single-leg hop cluster (hop for } \\
\text { distance, triple hop, crossover, } 6 \mathrm{~m} \\
\text { timed) }>80 \% \text { of uninvolved limb }\end{array}$ & $\begin{array}{l}\text { Quadriceps LSI }>90 \% \\
\text { · Hip abductors LSI }>95 \% \\
\text { · Hamstring LSI }>95 \% \\
\text { · Good neuromuscular control } \\
\text { with } 60 \text {-second timed step- } \\
\text { down test } \\
\text { - Good landing mechanics with } \\
60 \text { - second timed lateral leap } \\
\text { and catch } \\
\text { · Good landing mechanics with } \\
\text { single-leg hop cluster (hop for } \\
\text { distance, triple hop, crossover, } 6 \\
\text { m timed) }>90 \% \text { of uninvolved } \\
\text { limb } \\
\text { - Good acceleration, } \\
\text { deceleration, change of } \\
\text { direction control } \\
\text { · Ready to initiate controlled } \\
\text { contact }\end{array}$ & $\begin{array}{l}\text { Quadriceps LSI }>95 \% \\
\text { · Hip abductors LSI }>95 \% \\
\cdot \text { Hamstring LSI }>95 \% \\
\text { · Excellent neuromuscular } \\
\text { control with } 60 \text {-second } \\
\text { timed step-down test } \\
\text { · Excellent landing } \\
\text { mechanics with } 60 \text {-second } \\
\text { timed lateral leap and catch } \\
\text { · Excellent landing } \\
\text { mechanics with single-leg } \\
\text { hop cluster (hop for } \\
\text { distance, triple hop, } \\
\text { crossover, } 6 \text { m timed) } \\
>95 \% \text { of uninvolved limb } \\
\text { · Excellent acceleration, } \\
\text { deceleration, change of } \\
\text { direction control } \\
\text { - Comfortable with all } \\
\text { noncontact and controlled } \\
\text { contact drills } \\
\text { · Participated in reduced } \\
\text { practice, completing final } \\
\text { preparation for return to full } \\
\text { contact play }\end{array}$ \\
\hline Exercise recommendations & $\begin{array}{l}\text { Continue with progressive resisted } \\
\text { strengthening } \\
\text { · Maintain ROM and monitor } \\
\text { effusion } \\
\text { - Initiate return to jogging program } \\
\text { - Advanced plyometrics from } \\
\text { double to single leg, from simple to } \\
\text { complex } \\
\text { · Advance single leg perturbation } \\
\text { training } \\
\text { - Linear change in direction drills } \\
\text { - Lateral change in direction drills } \\
\text { - Sport-specific agility drills }\end{array}$ & $\begin{array}{l}\text { Running drills (straight line, } \\
\text { zigzag, rotation, change in } \\
\text { speed, change in direction) } \\
\text { - Rotational control drills } \\
\text { - Sport-specific drills: } \\
\text { noncontact drills only }\end{array}$ & $\begin{array}{l}\text { Initiate controlled contact } \\
\text { sport-specific drills: contact } \\
\text { drills, position-specific } \\
\text { training }\end{array}$ \\
\hline Cardiovascular recommendations & $\begin{array}{l}\text { Sport-specific cardiovascular } \\
\text { training } \\
\cdot \text { Longer runs (20-30 minutes in } \\
\text { duration) }\end{array}$ & $\begin{array}{l}\text { Interval training } \\
\cdot \text { Tempo runs } \\
\cdot \text { Fartleks } \\
\cdot \text { Shuttle runs }\end{array}$ & - Same as Phase 4 \\
\hline Pool program & Water jogging & $\begin{array}{l}\text { Water jogging } \\
\text { - Swimming can be used for } \\
\text { nonimpact cardiovascular } \\
\text { training, as deemed appropriate }\end{array}$ & $\begin{array}{l}\text { Swimming can be used for } \\
\text { nonimpact cardiovascular } \\
\text { training, as deemed } \\
\text { appropriate }\end{array}$ \\
\hline
\end{tabular}

LSI, limb symmetry index; ROM, range of motion.

While both categories of exercise appear to be beneficial toward optimizing strength, clinicians should consider these findings when selecting exercises after MPFC reconstruction. OKC exercises such as leg extension and long arc quad exercises appear to be more appropriate during greater degrees of knee flexion. CKC exercises, such as leg press, wall squat, forward lunge, lateral lunge, and split squat demonstrate less compressive stress when performed within fewer degrees of knee flexion. ${ }^{23-25}$ Zhang et al. performed a prospective study comparing the clinical effects of open and closed chain exercises after MPFL reconstruction. ${ }^{25}$ In their series of 40 participants, they found that the CKC exercise group performed better with the single-leg hop test, muscle atrophy, knee function, and pain reduction than the open kinetic chain group at 3, 6, and 12 months.

Early rehabilitation following MPFC reconstruction emphasizes decreasing effusion and postoperative pain along with improving mobility and reestablishing quadriceps control (Table 1). Many physicians will allow gradual increases in flexion over 
the first 6 weeks with recommendations of immediate weight bearing as tolerated with postoperative brace locked in extension for ambulation. Again, the presence of concurrent procedures, such as TTO, can change these recommendations and should be clearly identified at the outset. As the athlete progresses into the subsequent phases of rehabilitation, strengthening and neuromuscular training become the primary rehabilitation goal in preparation for impact activities. Prior to engaging in impact activities, it is expected that the athlete would have minimal to no effusion, full, symmetrical knee range of motion and quadriceps strength $>80 \%$ limb symmetry index (LSI). Along with these objective markers, it is also expected the athlete can demonstrate appropriate mechanics during a singleleg squat, including adequate depth, without significant knee valgus or hip internal rotation. When the athlete has met these criteria, which is typically seen in the 12-16 week postoperative timeframe, the athlete can then begin the walk-to-jog progression, as outlined by the physical therapist. A generalized protocol is detailed in Table 2 . Concomitant procedures, such as TTO, can contribute to a slower progression due to prolonged weight-bearing precautions or slower return of quadriceps strength. This may ultimately lead to a delayed RTS timeline, as some studies have reported longer RTS timeframe with TTO than those which did not. ${ }^{20,26}$

\section{Functional Testing and Return to Sport}

Evidence concisely outlining functional tests and RTS tests following MPFC reconstruction remains sparse. Return-to-play guidelines are not currently clearly defined, along with existing inconsistencies in rehabilitation protocols and variances of surgical procedures. ${ }^{27}$ A recent RTP systematic review showed that in a review of 39 articles from 1997 to 2019 , only 16 showed any specific criteria for RTS after patellofemoral surgery. ${ }^{21}$ Even when RTS tests were used, no studies stated explicitly the numerical values necessary for RTS. Coda et al. evaluated 38 publicly available protocols, and only 13 of the 38 protocols $(34.2 \%)$ included specific strength or functional testing guidelines for criteria to RTS. $^{28}$ Without clearly established return-to-play guidelines, athletes may be returning to sport with functional deficits that may increase injury risk or decrease ability to resume prior level of sport. Currently, RTS guidelines are most commonly seen within ACL literature. With similar injury mechanisms tests and postoperative neuromuscular deficiencies, it has been suggested that strategies used for RTS testing for ACL reconstruction can be applied to patellofemoral surgeries and used to provide feedback for the athlete and clinician. ${ }^{26,29}$

\section{Strength Testing}

In order to assess the readiness of an athlete for RTS progression, using a combination of tests can provide valuable information to both the athlete and clinician. Testing should include measures of strength, neuromuscular control, and balance. Quadriceps strength following MPFC reconstructions has shown persistent deficits at 6 months, and even up to 3 years, particularly with concomitant procedures such as TTO. ${ }^{20,21}$ Criteria for RTS in ACLR commonly used are $>90 \%$ with some arguing for $>95-100 \%$ given the expected deconditioning effects occurring in the contralateral limb. ${ }^{30}$ While limited evidence currently exists to support the relationship between isokinetic strength and rate of reinjury after MPFC reconstruction, strength testing can additionally provide insight into existing functional deficits that can be addressed with rehabilitation.

Isokinetic testing has long been considered a "gold standard" for strength testing of quadriceps and hamstrings. ${ }^{31}$ For strength testing, if access to an isokinetic testing is not available, using a hand-held dynamometer (HHD) has been shown to be a valid and reliable measure of strength. Quadriceps measurements can be performed in a seated-leg extension machine (hips at $90^{\circ}$ flexion and knees at $60^{\circ}$ of flexion) with HHD placed $5 \mathrm{~cm}$ above the medial malleolus along the anterior tibia. The athlete is asked to slowly extend their limb against the fixed machine and push into the device using maximal effort. Following a practice trial, the athlete performs 3 repetitions, and the average is calculated for each limb. Using the absolute value from each limb, the LSI is then calculated and can highlight deficiencies in the surgical limb. The test is also repeated for the hamstrings and performed in the prone position with the hip at $0^{\circ}$ of flexion and knee at $0^{\circ}$ of flexion. The same protocol as quadriceps testing is used, and LSI is calculated.

With the mechanism of patellofemoral injuries often occurring with increased dynamic knee valgus and hip internal rotation, identifying hip abduction deficits can be crucial in preventing future injuries. ${ }^{32}$ Testing for hip abduction strength can be performed in a side lying position with the testing limb in slight extension. The average of three attempts on each side can then be compared to determine an LSI with a goal of $>95 \%$. Hip strength deficits have been shown in athletes with patellofemoral dysfunction and may protect against lower extremity injuries. ${ }^{32,33}$

\section{Assessment of Motor Control}

Persistent motor control deficits have also been shown to contribute to "persistent kinesphobia". ${ }^{30}$ MPFC reconstruction with or without bony procedures can correct anatomical factors and bony abnormalities and improve static stability, but it does not address the motor control or dynamic stability that can 
Fig 1. The drop jump is a test using a 35-cm-high box. The athlete jumps off the box (A), lands on both feet, and immediately jumps back up off the ground, and then completes the test landing on both feet (B). The clinician assesses the athlete's control during acceleration and deceleration.
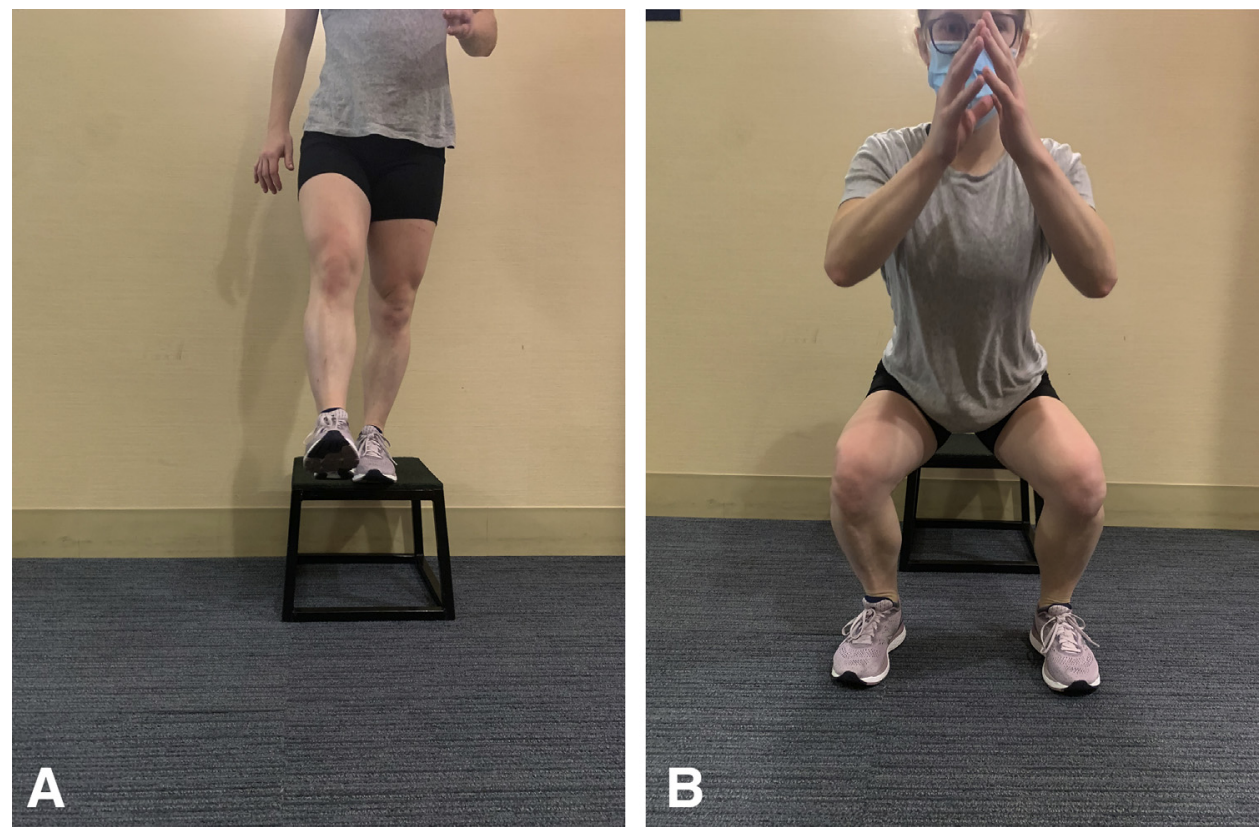

contribute to reinjury following reconstruction. In addition to strength testing, hop testing has been traditionally used in ACLR to identify asymmetries in function. The four tests commonly used-single-leg hop for distance, triple hop for distance, crossover hop for distance, and 6-m timed hop-are reliable and valid measures of testing. However, it remains crucial to evaluate the quality of motion along with distance hopped. The tests are performed as described by Noyes et al. ${ }^{34}$ While $>90 \%$ LSI assessing distance can be used as a guideline for RTS, it is important to note that LSI alone may not fully capture deficits in load absorption at the hip, knee, or ankle or sagittal/frontal planes asymmetries. It is also worth noting, similar to the strength testing, that the contralateral limb may also demonstrate mechanics that could increase injury risk of either limb, and should be considered when reporting strength as a percentage of the contralateral leg. ${ }^{30}$

Additional tests to assess quality of motion can include the side hop test, drop jump, lateral step-down test, and lateral leap and catch. ${ }^{35}$ The side hop test consists of jumping on one leg between two lines at a distance of $40 \mathrm{~cm}$ as many times as possible in $30 \mathrm{~s}$. Quality of motion, endurance, change of direction, and the number of hops are evaluated by the test. Drop jump (Fig 1) is a test that provides information on the quality of an athlete's landing. In the test, the patient drops from a box $35 \mathrm{~cm}$ high, lands on both feet, and immediately jumps as high as possible before the second landing. For both the lateral step-down test and lateral leap and catch, the clinician uses a metronome at 80 beats per minute (bpm) and $40 \mathrm{bpm}$, respectively. The lateral step-down test (Fig 2) is performed with the athlete standing on one limb at the edge of a box while performing a lateral step down to $60^{\circ}$ of flexion while tapping the heel of the contralateral limb to the ground. The timer is set for 3 minutes, as the clinician assesses for any "strikes" constituted by a loss of balance, significant trunk lean or knee valgus, and inability to match the beat of the metronome. When the athlete has demonstrated 3 strikes or reports inability to continue testing, the clinician marks the time. For the lateral leap and catch (Fig 3), a timer is set for 60 seconds as the athlete hops laterally between limbs a

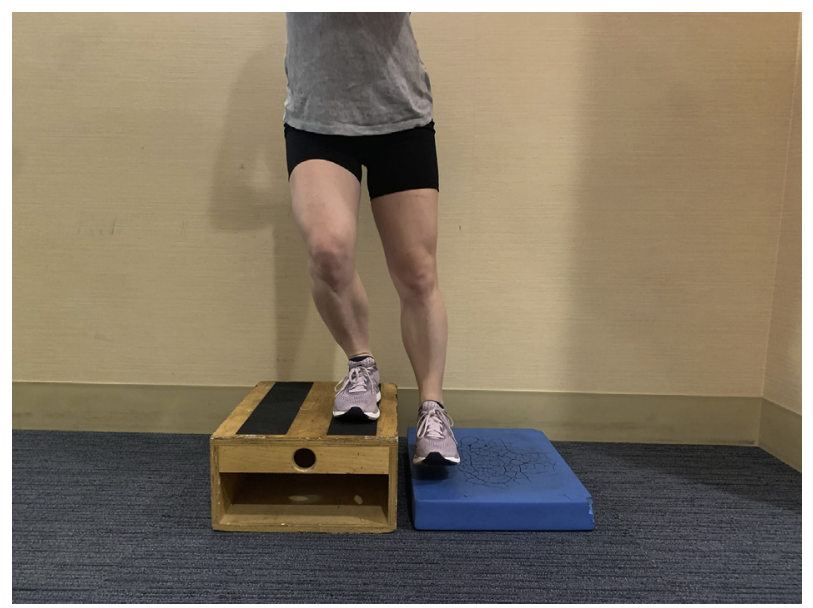

Fig 2. The lateral step-down test is a 3-minute timed test. The athlete stands on one limb at the edge of a box while performing a lateral step-down to $60^{\circ}$ of knee flexion, tapping the heel of the contralateral limb to the ground, at a beat of 80 beats per minute (bpm). The clinician assesses for any loss of balance or aberrant movement patterns. 

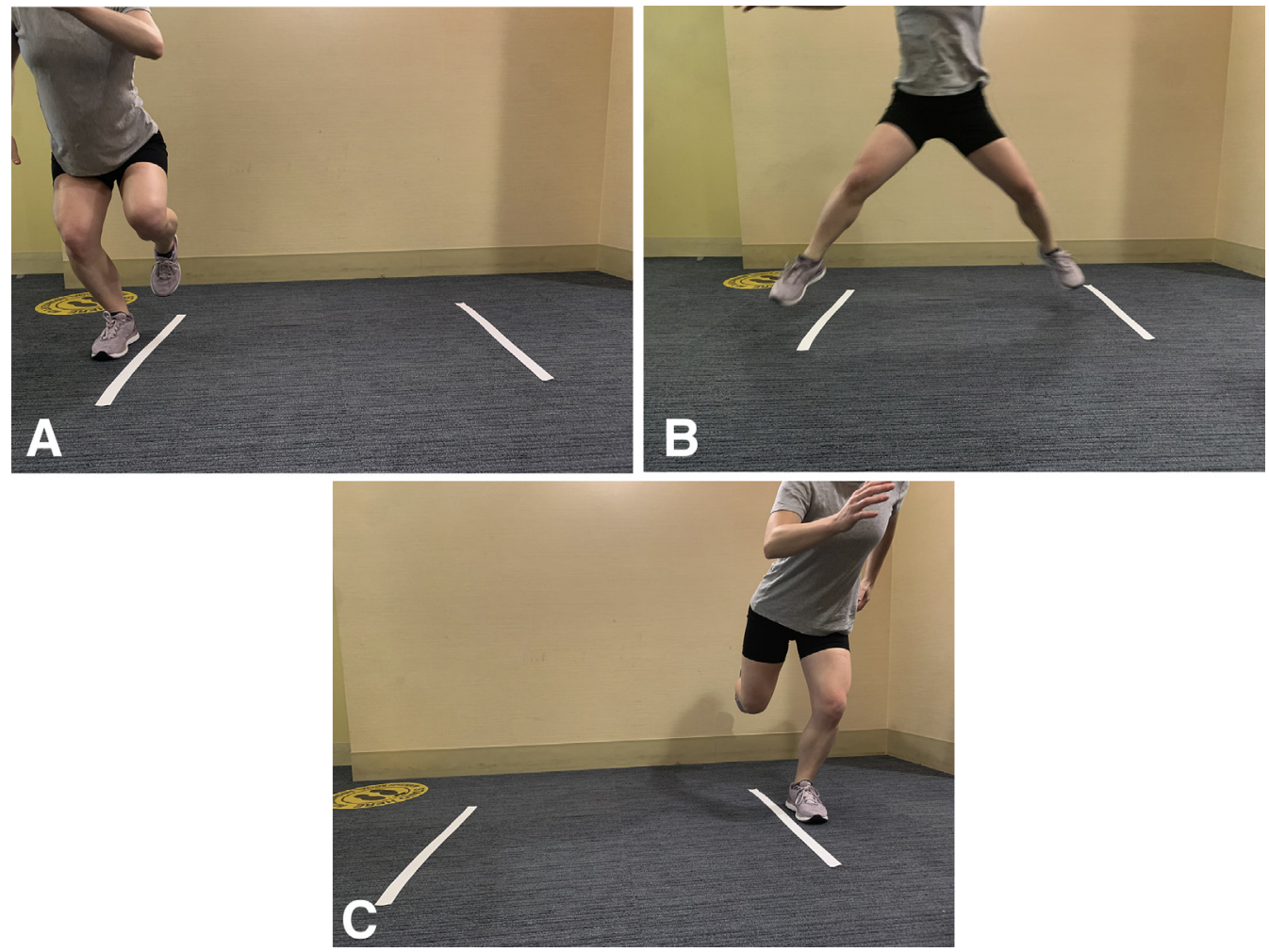

Fig 3. The lateral leap and catch is a 60 -s test. The athlete hops laterally from one limb to the other to a beat of $40 \mathrm{bpm}(\mathrm{A}-\mathrm{C})$. The distance between the hops should be $60 \%$ of the athlete's height. The clinician monitors for neuromuscular control and overall shock attenuation with change in direction. distance set at $60 \%$ of their height as the clinician assesses quality of motion and ability to match the beat of the metronome.

Poor dynamic balance has been considered an intrinsic risk for injuries in athletes and should be assessed and corrected prior to clearance for RTP. For assessment of single-leg dynamic stability, the Y-balance test (YBT), which has been shown to have good inter-rater and intrarater reliability and has been shown to be a predictor of lower-extremity injury risk when there is $>4 \mathrm{~cm}$ deficit in the forward reach or $<90 \%$ composite score, which incorporates the leg length in its calculation. ${ }^{36}$ During the YBT, the athlete is asked to stand on a central platform with toes at a designated line and uses the contralateral limb to push a block as far as possible with control and the ability to resume starting position without loss of balance. The athlete should also maintain heel contact and avoid kicking the block forward. The furthest distance is calculated in the anterior, mediolateral, and posterolateral positions and then repeated on opposite limb. ${ }^{36}$

\section{Subjective Measures}

Fear of reinjury has been reported by athletes as a common reason not to return to sport. ${ }^{37}$ In addition to the performance measures commonly used, it is also valuable to use patient-reported outcome measures (PROMs) of different constructs to assess subjective readiness of the athlete. Commonly used PROMs after MPFC reconstruction have included the Kujala score, IKDC, Lysholm, Tegner, and Banff Patella Instability Index. ${ }^{38}$ In addition to these subjective measures of performance, it is also critical to evaluate the athlete's confidence in returning back to sport. The anterior cruciate ligament return to sport after injury (ACL-RSI) measure, commonly used after ACL surgery, has shown predictive value in RTS at 4 months and has also gone on to demonstrate a second injury risk when scoring $<70 .{ }^{37,39}$ Along with the ACL-RSI, the Tampa Scale of Kinesiophobia (TSK-11) can also be an important tool in determining an athlete's fear of movement when returning back to sport. Shah et al. reported that $32 \%$ of athletes described apprehension prior to RTS. The TSK-11 is an 11 -item self-administered questionnaire aimed at quantifying the fear of reinjuries due to movement and physical activity. ${ }^{40}$ With fear of "reinjury" as the primary reason for not returning to previous level of play after both ACL and MPFL reconstruction, parallels can be drawn when considering value of confidence scores with RTS. High motivation and low fear have also been shown to positively correlate with greater rates of return to play. ${ }^{37}$

For each athlete, the decision-making progress for RTS should be multifactorial and encompass objective and subjective criteria from testing, discussions with the referring surgeon, and an appropriate return to sport 
progression. The type of sport and demands associated with each sport should also be considered prior to full clearance of activity. For the tests described above, it has been demonstrated that athletes who are not "passing" at 6 months may require additional rehabilitation to improve any deficiencies noted. ${ }^{27}$ After an athlete passes a series of RTS tests, they can begin a progression of independent sport-specific activities in a controlled environment, into unpredictable settings at faster speeds ( $1 \mathrm{vl}>3 \mathrm{v} 3$ drills). As the athlete gains confidence in the uncontrolled environment, light contact may be introduced, progressing into full scrimmages and lastly full competitive match. The goals for each of these phases is to allow for appropriate increases in endurance, confidence at increasing speeds, and the ability to react in unpredictable environments. If the athlete were to have any persistent symptoms during these phases, the athlete should remain at their current level of activity or return to the prior phase if there is no resolution of symptoms.

\section{Conclusion}

The decision to progress an athlete through the return to sport phases should be based on criteria achieved and quality of motion, not solely based on time, with considerations of the specifics of the patellofemoral joint for both rehabilitative and preventative training purposes. With a large percentage of athletes returning to sport following MPFC reconstruction, there is a need for validated consensus regarding RTP guidelines. Using standardized guidelines may be helpful in decreasing reinjury rates, improving patient outcomes, and setting realistic patient expectations. It is important to assess any sport-specific maneuvers along with their subjective report of function and confidence in returning to their sport, in order to optimize return to play outcomes after MPFC reconstruction.

\section{References}

1. Sillanpää P, Mattila VM, Iivonen T, Visuri T, Pihlajamäki H. Incidence and risk factors of acute traumatic primary patellar dislocation. Med Sci Sports Exerc 2008;40:606-611.

2. Duthon VB. Acute traumatic patellar dislocation. Orthop Traumatol Surg Res 2015;101:S59-S67.

3. Hsiao M, Owens BD, Burks R, Sturdivant RX, Cameron KL. Incidence of acute traumatic patellar dislocation among active-duty United States military service members. Am J Sports Med 2010;38:1997-2004.

4. Jaquith BP, Parikh SN. Predictors of recurrent patellar instability in children and adolescents after first-time dislocation. J Pediatr Orthop 2017;37:484-490.

5. Conlan T, Garth WP Jr, Lemons JE. Evaluation of the medial soft-tissue restraints of the extensor mechanism of the knee. J Bone Joint Surg Am 1993;75:682-693.

6. Smirk C, Morris H. The anatomy and reconstruction of the medial patellofemoral ligament. Knee 2003;10:221-227.
7. Hautamaa PV, Fithian DC, Kaufman KR, Daniel DM, Pohlmeyer AM. Medial soft tissue restraints in lateral patellar instability and repair. Clin Orthop Relat Res 1998;349:174-182.

8. Fulkerson JP, Edgar C. Medial quadriceps tendon-femoral ligament: surgical anatomy and reconstruction technique to prevent patella instability. Arthrosc Tech 2013;2: el25-el28.

9. Placella G, Tei M, Sebastiani E, et al. Anatomy of the medial patello-femoral ligament: A systematic review of the last 20 years literature. Musculoskelet Surg 2015;99: 93-103.

10. Mochizuki T, Nimura A, Tateishi T, Yamaguchi K, Muneta T, Akita K. Anatomic study of the attachment of the medial patellofemoral ligament and its characteristic relationships to the vastus intermedius. Knee Surg Sports Traumatol Arthrosc 2013;21:305-310.

11. Tanaka MJ. Variability in the patellar attachment of the medial patellofemoral ligament. Arthroscopy 2016;32: 1667-1670.

12. Tanaka MJ, Voss A, Fulkerson JP. The anatomic midpoint of the attachment of the medial patellofemoral complex. J Bone Joint Surg Am 2016;98:1199-1205.

13. Tanaka MJ, Chahla J, Farr J 2nd, et al. Recognition of evolving medial patellofemoral anatomy provides insight for reconstruction. Knee Surg Sports Traumatol Arthrosc 2019;27:2537-2550.

14. Spang RC, Tepolt FA, Paschos NK, Redler LH, Davis EA, Kocher MS. Combined reconstruction of the medial patellofemoral ligament (MPFL) and medial quadriceps tendon-femoral ligament (MQTFL) for patellar instability in children and adolescents: Surgical Technique and Outcomes. J Pediatr Orthop 2018;39:e54-e61.

15. Placella G, Tei MM, Sebastiani E, et al. Shape and size of the medial patellofemoral ligament for the best surgical reconstruction: a human cadaveric study. Knee Surg Sports Traumatol Arthrosc 2014;22:2327-2333.

16. Enderlein D, Nielsen T, Christiansen SE, Fauno P, Lind M. Clinical outcome after reconstruction of the medial patellofemoral ligament in patients with recurrent patella instability. Knee Surg Sports Traumatol Arthrosc 2014;22: 2458-2464.

17. Redler LH, Meyers KN, Brady JM, Dennis ER, Nguyen JT, Shubin Stein BE. Anisometry of medial patellofemoral ligament reconstruction in the setting of increased tibial tubercle-trochlear groove distance and patella alta. Arthroscopy 2018;34:502-510.

18. Stephen JM, Dodds AL, Lumpaopong P, Kader D, Williams A, Amis AA. The ability of medial patellofemoral ligament reconstruction to correct patellar kinematics and contact mechanics in the presence of a lateralized tibial tubercle. Am J Sports Med 2015;43:2198-2207.

19. Manjunath AK, Hurley ET, Jazrawi LM, Strauss EJ. Return to play after medial patellofemoral ligament reconstruction: A systematic review. Am J Sports Med 2021;49: 1094-1100.

20. Krych AJ, O'Malley MP, Johnson NR, et al. Functional testing and return to sport following stabilization surgery for recurrent lateral patellar instability in competitive athletes. Knee Surg Sports Traumatol Arthrosc 2018;26: 711-718. 
21. Chatterji R, White AE, Hadley CJ, Cohen SB, Freedman KB, Dodson CC. Return-to-play guidelines after patellar instability surgery requiring bony realignment: A systematic review. Orthop J Sports Med 2020;8: 2325967120966134.

22. Matassi F, Innocenti M, Andrea CL, et al. Timing for safe return to sport after medial patellofemoral ligament reconstruction: The role of a functional test battery. J Knee Surg 2021;34:363-371.

23. Escamilla RF, Zheng N, MacLeod TD, et al. Patellofemoral compressive force and stress during the forward and side lunges with and without a stride. Clin Biomech (Bristol, Avon) 2008;23:1026-1037.

24. Escamilla RF, Fleisig GS, Zheng N, Barrentine SW, Wilk KE, Andrews JR. Biomechanics of the knee during closed kinetic chain and open kinetic chain exercises. Med Sci Sports Exerc 1998;30:556-569.

25. Zhang F, Wang J, Wang F. Comparison of the clinical effects of open and closed chain exercises after medial patellofemoral ligament reconstruction. J Phys Ther Sci 2014;26:1557-1560.

26. Ménétrey J, Putman S, Gard S. Return to sport after patellar dislocation or following surgery for patellofemoral instability. Knee Surg Sports Traumatol Arthrosc 2014;22: 2320-2326.

27. Lieber AC, Steinhaus ME, Liu JN, Hurwit D, Chiaia T, Strickland SM. Quality and variability of online available physical therapy protocols from academic orthopaedic surgery programs for medial patellofemoral ligament reconstruction. Orthop J Sports Med 2019;7: 2325967119855991.

28. Coda RG, Cheema SG, Hermanns C, et al. Online rehabilitation protocols for medial patellofemoral ligament reconstruction with and without tibial tubercle osteotomy are variable among institutions. Arthrosc Sports Med Rehabil 2021;3:e305-e313.

29. Fithian DC, Powers CM, Khan N. Rehabilitation of the knee after medial patellofemoral ligament reconstruction. Clin Sports Med 2010;29:283-290. ix.

30. Wellsandt E, Failla MJ, Snyder-Mackler L. Limb symmetry indexes can overestimate knee function after anterior cruciate ligament injury. J Orthop Sports Phys Ther 2017;47: 334-338.

31. Stark T, Walker B, Phillips JK, Fejer R, Beck R. Hand-held dynamometry correlation with the gold standard isokinetic dynamometry: A systematic review. PM R 2011;3: 472-479.
32. Leetun DT, Ireland ML, Willson JD, Ballantyne BT, Davis IM. Core stability measures as risk factors for lower extremity injury in athletes. Med Sci Sports Exerc 2004;36: 926-934.

33. Baldon Rde M, Nakagawa TH, Muniz TB, Amorim CF, Maciel CD, Serrão FV. Eccentric hip muscle function in females with and without patellofemoral pain syndrome. J Athl Train 2009;44:490-496.

34. Noyes FR, Barber SD, Mangine RE. Abnormal lower limb symmetry determined by function hop tests after anterior cruciate ligament rupture. Am J Sports Med 1991;19: 513-518.

35. Haitz K, Shultz R, Hodgins M, Matheson GO. Test-retest and interrater reliability of the functional lower extremity evaluation. J Orthop Sports Phys Ther 2014;44: 947-954.

36. Plisky PJ, Gorman PP, Butler RJ, Kiesel KB, Underwood FB, Elkins B. The reliability of an instrumented device for measuring components of the star excursion balance test. N Am J Sports Phys Ther 2009;4: 92-99.

37. Ardern CL, Taylor NF, Feller JA, Whitehead TS, Webster KE. Psychological responses matter in returning to preinjury level of sport after anterior cruciate ligament reconstruction surgery. Am J Sports Med 2013;41: 1549-1558.

38. Collins NJ, Misra D, Felson DT, Crossley KM, Roos EM. Measures of knee function: International Knee Documentation Committee (IKDC) Subjective Knee Evaluation Form, Knee Injury and Osteoarthritis Outcome Score (KOOS), Knee Injury and Osteoarthritis Outcome Score Physical Function Short Form (KOOS-PS), Knee Outcome Survey Activities of Daily Living Scale (KOS-ADL), Lysholm Knee Scoring Scale, Oxford Knee Score (OKS), Western Ontario and McMaster Universities Osteoarthritis Index (WOMAC), Activity Rating Scale (ARS), and Tegner Activity Score (TAS). Arthritis Care Res (Hoboken) 2011;63:S208-S228 (Suppl 11).

39. McPherson AL, Feller JA, Hewett TE, Webster KE. Psychological readiness to return to sport is associated with second anterior cruciate ligament injuries. Am J Sports Med 2019;47:857-862.

40. Shah JN, Howard JS, Flanigan DC, Brophy RH, Carey JL, Lattermann C. A systematic review of complications and failures associated with medial patellofemoral ligament reconstruction for recurrent patellar dislocation. Am J Sports Med 2012;40:1916-1923. 